\title{
Applied Study on E-Marketing Websites with Designing Home Delivery Service Website
}

\author{
Mohammed Refai ${ }^{1}$, Mhmmad Al_sonbati ${ }^{2}$, Sawsan Houdaly ${ }^{3}$, Ghadeer Mallah ${ }^{4}$ \\ Professor, Department of Software Engineering, IT College, Zarqa University, Jordan 1 \\ Student, Department of Software Engineering, IT College, Zarqa University, Jordan ${ }^{2,3,4}$
}

\begin{abstract}
The main idea in this research is to design an E-Marketing website that deal with the problems in previous sites. Top ten sites in E-Marketing according to the most frequently used search engines "Google and Yahoo" are visited and evaluated according to certain criteria. Advantages and drawbacks are extracted and compared. Researchers built Home delivery website that avoid many negative points in the analyzed sites.
\end{abstract}

Keywords: Include at least 4 keywords or phrases

\section{INTRODUCTION}

E-commerce is a new term in the online world. It makes buying and selling, transferring funds, advertising and displaying products easy. E-commerce is a creative idea as it allows companies to offer their goods and products through their websites [2, 5]. E-marketing means using digital technologies such as websites, mobile devices and social networking to help reach your customer base, create awareness of your brand and sell your goods or services. These technologies can be used cheaply and effectively, whatever the size of your company or your business model. [1, 2]

The basics of marketing remain the same - creating a strategy to deliver the right messages to the right people. Though business will continue make use of traditional marketing methods, such as advertising and direct mail. Emarketing adds a whole new element to the marketing mix. Its flexible and cost-effective nature makes it particularly suitable for small businesses.

E-marketing gives businesses of any size access to the mass market at an affordable price and, unlike TV or print advertising, it allows truly personalized marketing. Specific benefits of e-marketing include: Global reach - a website allows you to find new markets and trade globally for only a small investment. Lower cost - a properly planned and effectively targeted e-marketing campaign can reach the right customers at a much lower cost than traditional marketing methods.

Work reported here is aiming to present a comprehensive survey of e-marketing and delivery service to homes websites. Researchers use top ten sites according to the most frequently use search engines "Google and Yahoo". Evaluation of these sites according to certain criteria is made to extract advantages and drawbacks. A web site about electronic marketing and delivery service to homes was built with avoidance of many negative points that were found at the sites analyzed. The new web site helps customers to shop and use the home delivery services so they can save a lot of time and effort. Customers can use the internet to buy what they need from this website while setting home, simply by logging in to our page and choosing what they want. Then the delivery employee delivers the product to the customer's home and gets the money from the customer. Designed web site focused on security and safety issues for customers money and market rights.

\section{RELATED WORK}

In [7] This paper presents a framework of website quality evaluation for measuring the performance of government websites. Multiple criteria decision-making (MCDM) is a widely used tool for evaluating and ranking problems containing multiple, usually conflicting criteria. In line with the multi-dimensional characteristics of website quality, MCDM provides an effective framework for an inter-websites comparison involving the evaluation of multiple attributes. It thus ranks different websites compared in terms of their overall performance. This paper models the inter-website comparison problem as an MCDM problem, and presents a practical and selective approach to deal with it. In addition, fuzzy logic is applied to the subjectivity and vagueness in the assessment process. The proposed framework is effectively illustrated to rate Turkish government websites.[7]

In [8] The Content Management Systems (CMS) are widely used on the Internet. They separate the content creation from the site design. Users without having web design or programming knowledge can create a professional website using a ready-made application that allows them to concentrate only over the content creation. A site powered by a CMS is called a dynamic website, because its content (text, images, and animations) could respond in various ways to the visitors' requests. The dynamic content is created using client site programming (JavaScript for example) or server side programming (ASP, PHP). Static websites are made only from HTML files.

In order to modify them you must edit one by one, Usually CMS are database driven. The visitors will look at 
the front end, which is the final website displaying your content, while the backend allows you to manage the content that is stored in a database.

Dynamic sites are easy to update and maintain. When you deal only with static html pages, the updating process could take hours, while the use of a CMS will simplify the content editing process. This is performed from an administration panel through a simple browser based interface. Another advantage of CMS is the increased interactivity and functionality of your website. Most of CMS have lots of modules that allow you to enhance your website in minutes, website development being faster as compared to creation of static html files. For example you can have a news publishing system, a forum, a blog and an image gallery on the same website due to the CMS modularity. The disadvantages of CMS over static websites (formed only by HTML pages): low pages loading speed on client side due to multiple database requests and the SEO (search engine optimization) process is not always optimal due to the web addresses format, because the pages address names are dynamically generated. These impediments can be eliminated by optimizing CMS using PHP cache optimizers and short URL's names generators. The best practice is to carefully decide when a CMS is necessary, depending of the website size, the time interval when the content is updated and the web hosting services details.

In [14] Author presents six criteria for a thorough evaluationfounded type of business, purpose of its website etc. after identifying a number of potential web sources. TheseInformation about the products adequate information selection and evaluation criteria are the following: Visualabout the products is displayed, eg. Description, clues, authority, currency, relevance, accuracy, andphotographs, availability, prices etc objectivity.

\section{III.CRITERIA OF WeB Sit ANALySIS}

Researchers access to most popular websites in ecommerce and e-marketing and analyzed them by a set of the following criteria:

\section{- Architecture and Navigation}

Consistency: Page layout or style is consistence throughout the website, eg. justification of text, font types , font size, position of the navigation menu in each page .colures are consistent and provide consistent look and feel for navigation and information design ,eg. font colures ,background colures ,use of standard link colures (standard ,blue link colour should be used for unvisited pages and purple or red colours for visited pages ).

Navigation on Support Navigational links are obvious in each page so that users can explore and find their way around the site and navigate easily, e.g. index ,or site map, or navigation bar or table of contents.

Internal search: Internal search is effective, eg. Fast; accurate; provides useful, concise and clear results which are easy for interpreting.

Working Links: Links are discernible, working properly and not misleading so that the user knows what to expect from the destination pages, eg. Links are obvious, no broken links, link names match page names.
Resourceful The site is informative and resourceful; e.g. has links to external useful resources

No orphan pages The site has no dead-end pages, eg. Its easy and obvious to go to the home page from any subpage of the site .pages have a clear indication of their position within the site.

Logical structure of site The structure of the site is simple and straightforward, related information is grouped together, categorization of products is helpful.

Architecture is not too deep so that the number of click to reach goals is not too large.

Simple navigation menu Navigation menu is simple and straightforward, the menu choices are ordered logically so it is easy to understand the website.

\section{Content:}

Up-to-date information The information is up-to-date, current, often update, date of last update is displayed and informs the user when new information is added.

Relevant information The information is sufficient and relevant to user needs, e.g. content is concise and nonrepetitive, terminology/terms are clear and unambiguous, there are no under construction pages

Accurate Information The information is precise, e.g. product measurements, total price services, etc

Grammatical Accuracy Content is free from grammatical errors, eg. no spelling errors ,no grammar errors ,punctuation is accurate.

Information About the company Basic facts about the company or company overview are displayed, eg. Year

\section{Accessibility and communication:}

Easy to find and access website

The site is easily identifiable and accessible from search engines, the URL is domain related, not complex , and easy to remember .download time of the pages is appropriate.

Contact us information Useful information to enable easy communication with the company is displayed, e.g. .faq, contact us (eg. name, physical address, telephone number, fax number, email details), customer feedback form to submit customers comments .

Help lcustomer service The help lcustomer service is easy to find , has a clear and distinct layout ,searching for help lcustomer service is easy navigating in helplcustomer service is easy ,amount of information is sufficient ,concise and designed to answer the specific questions users will have in a specific context

Compatibility The site works with different browsers and on different monitor resolutions.

-Design:

Aesthetic design The site is attractive and appealing so that it impresses the potential customer.

Appropriate use of images Quality of images is adequate, no broken images, images make a contribution to the understanding and navigation of the site, alternative text is used for images, image size is relevant so that it has minimal effect on loading time. 
Appropriate choice of fonts and colours Font types are appropriate and easy to read. Choice of colures for both fonts and background is appropriate, combination of background and font colures is appropriate.

Appropriate page design Pages are uncluttered; headings are clear page margins are sufficient, minimum or no long pages with excessive white space that force scrolling; particularly on the home page of the website, page title is appropriate, describing the company name and contents of each page.

\section{- Transactions}

Easy order process Registration on site is easy, changing customer information is easy, logging on to the site is easy, ordering process is easy, changing the contents of the shopping cart(adding, deleting or editing) is easy obvious and accurate.

Ordering information Complete information about ordering is displayed and can be accessed easily, e.g. how to order, payment options, cancelling an order, return and refund policy, terms and conditions. Delivery information: Information about delivery and dispatch of an order is displayed, e.g. delivery times, delivery costs, delivery areas delivery address options (the ability to deliver the order to another address), delivery options, problems (e.g. non-delivery, late delivery, incorrect delivery address etc.)

Order\ delivery status provision Company informs the customer about order status, e.g. by sending confirmation email to customer after placing an order, by sending dispatch confirmation email to customer when order is sent out, by using online order tracking system. Alternative methods of ordering Ipayment Idelivery are available Alternative methods of ordering (e.g. online )payment (visa ,credit card ), cash on delivery ,cherub by boost ,bank transference) and delivery (standard ,express ,etc)are supported so that the user can select the method that suits him lher. Reasonable confidence in security and privacy The site uses secure socket layer or recognized secure payment methods, information about security guarantee and privacy policy is clearly displayed. Easy order process Registration on site is easy, changing customer information is easy, logging on to the site is easy, ordering process is easy, changing the contents of the shopping cart(adding, deleting or editing) is easy obvious and accurate .

Ordering information Complete information about ordering is displayed and can be accessed easily, e.g. how to order, payment options, cancelling an order, return and refund policy, terms and conditions.

Delivery information: Information about delivery and dispatch of an order is displayed, e.g. delivery times, delivery costs, delivery areas delivery address options (the ability to deliver the order to another address ), delivery options, problems (e.g. non-delivery, late delivery ,incorrect delivery address etc.)

Order $\backslash$ delivery status provision Company informs the customer about order status, e.g. by sending confirmation email to customer after placing an order, by sending dispatch confirmation email to customer when order is sent out, by using online order tracking system.

Alternative methods of ordering \payment \delivery are available. Alternative methods of ordering (e.g. online )payment (visa ,credit card ),cash on delivery ,cherub by boost ,bank transference) and delivery (standard, express ,etc)are supported so that the user can select the method that suits him \her .

Reasonable confidence in security and privacy The site uses secure socket layer or recognized secure payment methods, information about security guarantee and privacy policy is clearly displayed.

\section{IV.WEB SITS ANALYSIS}

Researchers list three websites in this section, and analysed them depended on the previous criteria, same idea was applied to the other seven sites to get advantages and treat all the drawbacks to obtain a comprehensive website:

1- $\quad$ www.mysupermarket.com

My Supermarket is a completely FREE website which allows you to compare supermarket prices as you shopping and get the best possible deal for your groceries figure 1 present the first page of this site.

Architecture and Navigation:

Exploration Heuristic

Page layout or style is consistence throughout the website, eg. justification of text, font types, font size .position of the navigation menu in each page . Colures are consistent and provide consistent look and feel for navigation and information design ,eg.font colures ,background colures ,use of standard link colures (standard ,blue link color should be used for unvisited pages and purple or red colors for visited pages ).

Consistency Navigational links are obvious in each page so that users can explore and find their way around the site and navigate easily ,e.g. index ,or site map, or navigation bar or table of contents.

Navigation on Support Internal search is effective, eg. fast ;accurate; provides useful, concise and clear results which are easy for interpreting

Internal search Links are discernible, working properly and not misleading so that the user knows what to expect from the destination pages, eg. links are obvious, no broken links, link names match page names .

Working Links The site is informative and resourceful, eg. it has links to external useful resources

Resourceful links The site has no dead-end pages ,eg. Its easy and obvious to go to the home page from any subpage of the site pages have a clear indication of their position within the site.

No orphan pages The structure of the site is simple and straightforward, related information is grouped together, categorization of products is helpful.

Architecture is not too deep so that the number of click to reach goals is not too large.

Logical structure of site Navigation menu is simple and straightforward, the menu choices are ordered logically so it is easy to understand the website. 


\section{Simple navigation menu}

Content:

Up-to-date information The information is up-to-date, current, often update ,date of last update is displayed and informs the user when new information is added

Relevant information The information is sufficient and relevant to user needs, e.g. content is concise and nonrepetitive, terminology/terms are clear and unambiguous, there are no under construction pages

Accurate Information The information is precise, e.g. product measurements, total price services, etc
Design:

Aesthetic design The site is attractive and appealing so that it impresses the potential customer .

Appropriate use of images Quality of images is adequate , no broken images, images make a contribution to the understanding and navigation of the site, alternative text is used for images, image size is relevant so that it has minimal effect on loading time.

Appropriate choice of fonts and colors Font types are appropriate and easy to read. Choice of colures for both fonts and background is appropriate, combination of

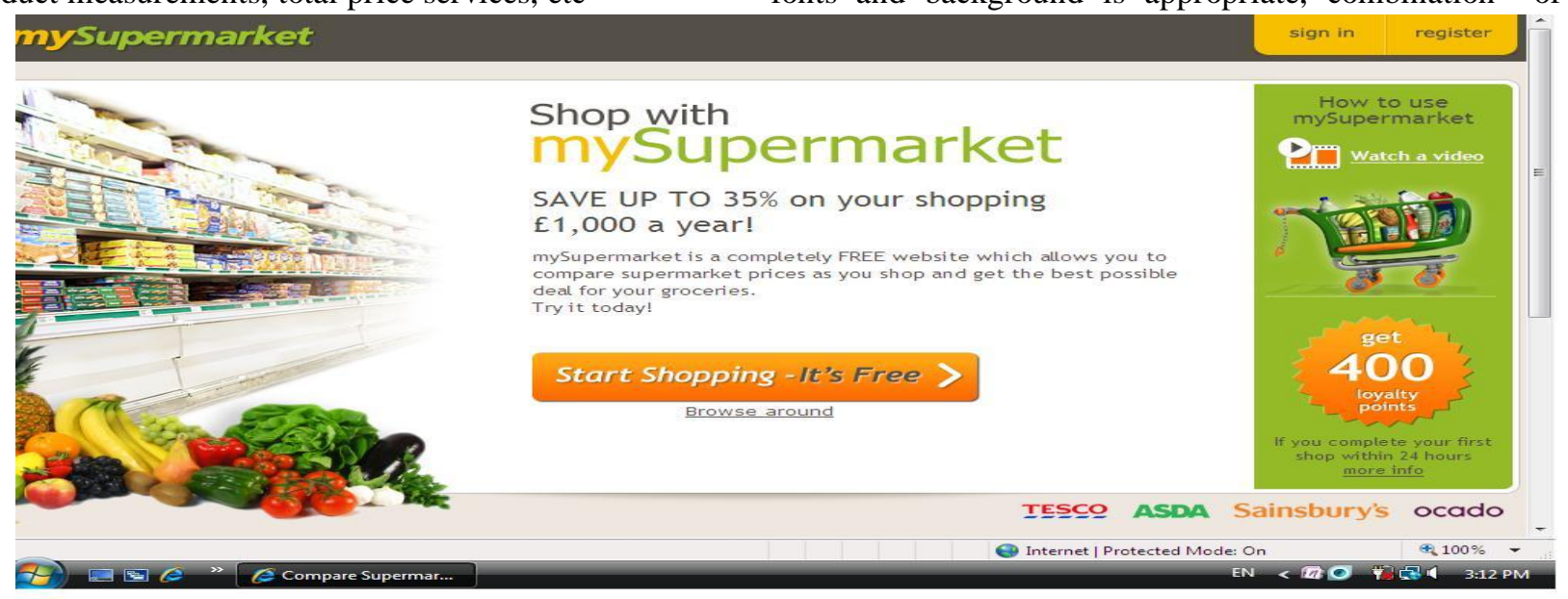

Fig-1 My Supermarket website

Grammatical Accuracy Content is free from grammatical background and font colures is appropriate .

errors, eg. No spelling errors, no grammar errors, Appropriate page design Pages are uncluttered, headings punctuation is accurate.

Information About the company Basic facts about the company or company overview are displayed ,eg. year founded, type of business, purpose of its website etc.

Information about the products Adequate information about the products is displayed ,eg. Description, photographs, availability, prices etc

Content:

Easy to find and access website The site is easily identifiable and accessible from search engines, the url is domain related, not complex, and easy to remember .download time of the pages is appropriate.

Contact us information Useful information to enable easy communication with the company is displayed, e.g. faq,contact us (eg.name, physical address, telephone number, fax number, email details), customer feedback form to submit customers comments .

Help lcustomer service The help lcustomer service is easy to find ,has a clear and distinct layout, searching for help lcustomer service is easy navigating in helplcustomer service is easy, amount of information is sufficient ,concise and designed to answer the specific questions users will have in a specific context.

Compatibility The site works with different browsers and on different monitor resolutions.

are clear page margins are sufficient, minimum or no long pages with excessive white space that force scrolling; particularly on the home page of the website, page title is appropriate, describing the company name and contents of each page.

\section{Transactions:}

Easy order process Registration on site is easy, changing customer information is easy, logging on to the site is easy, ordering process is easy, changing the contents of the shopping cart(adding, deleting or editing) is easy obvious and accurate .

Ordering information Complete information about ordering is displayed and can be accessed easily ,e.g. how to order, payment options ,cancelling an order, return and refund policy ,terms and conditions .

Delivery information Information about delivery and dispatch of an order is displayed, e.g. delivery times, delivery costs, delivery areas delivery address options (the ability to deliver the order to another address ), delivery options , problems (e.g. non-delivery, late delivery ,incorrect delivery address etc.)

Order $\backslash$ delivery status provision Company informs the customer about order status, e.g. by sending confirmation email to customer after placing an order, by sending dispatch confirmation email to customer when order is Foreign language and currency support The sites sent out, by using online order tracking system. content is displayed in different languages and uses more than one currency.

Alternative methods of ordering \payment Idelivery are available Alternative methods of ordering (e.g. online 
)payment (visa ,credit card ), cash on delivery, cherub by Working links very clear \& link match page name. boost ,bank transference) and delivery (standard ,express Resourceful link the site is not informative it has no link to ,etc)are supported so that the user can select the method external useful resources.

that suits him ther .

No orphan page: there is a orphan page because easy to Reasonable confidence in security and privacy The site move in the pages Logical structure of site.

uses secure socket layer or recognized secure payment Categorization of product is helpful; the architecture is not methods, Information about security guarantee and privacy deep so that the number of click to reach goals not large. policy is clearly displayed.

2- www.wairose.com : the second website to be analyzed is wairose which represents a Mall site

Use simple navigation menu. Description; photograph ; prices

Accessibility \&communication: Menu is Simple \&choices are ordered, easy to find $\&$ access web site

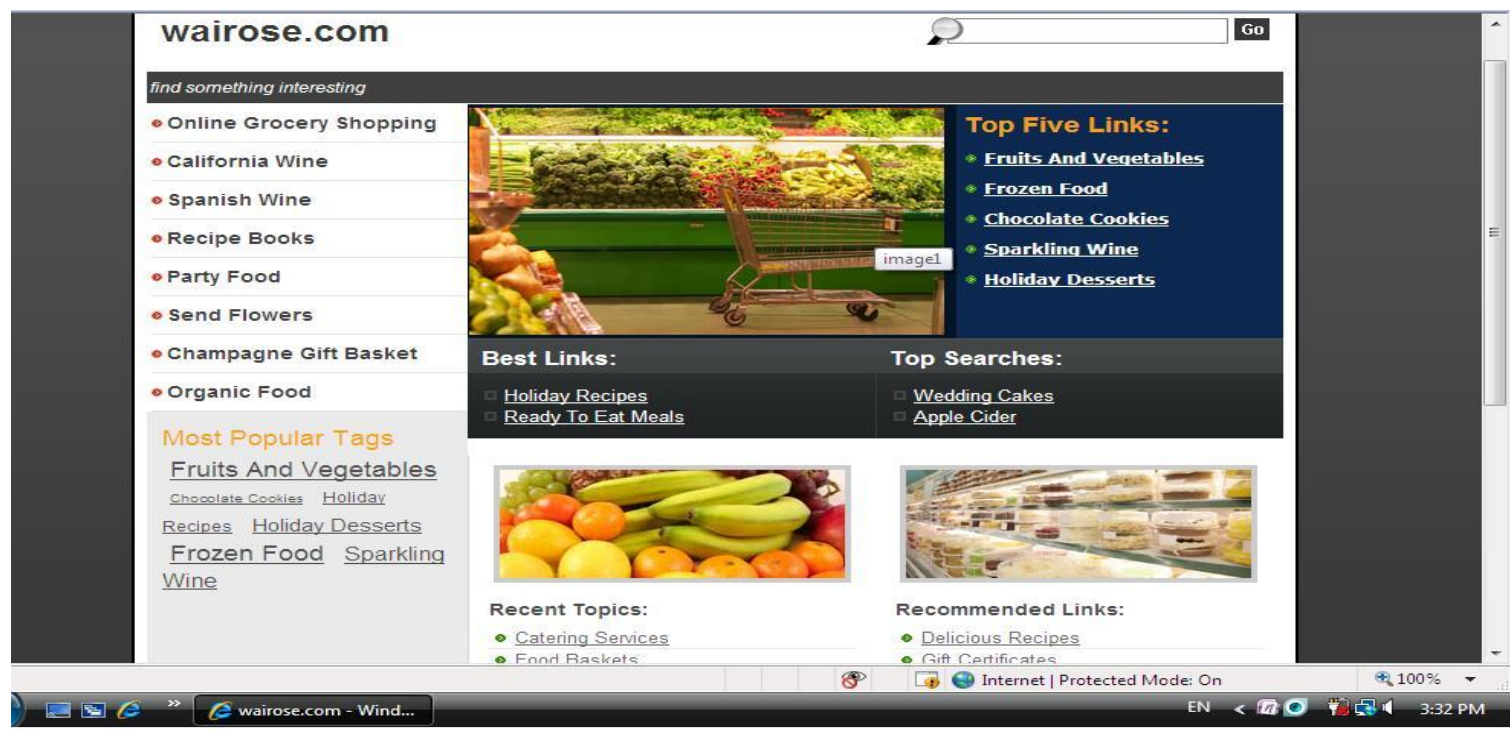

Figure-2 Wairose website

Description the site:- this The following analyses focus on advantages and disadvantages of this site.

\section{Architecture \&navigation}

Content: -Up-to-date information Often update is displayed \& informs the user when new information is added.

Relevant information Content is concise \& non repetitive. Information is precise; total price; services Grammatical Accuracy: Very good Information. About the company: overview was displayed and Information about the product.

Consistency Use more than colure of the key; background Page are cluttered; heading are not clear white \& black; good font size. so page layout \&style is consistent throughout the web sit

Navigation support Use index; \& use table of content; no site map; no navigation bar; Internal search is not understood \& very slow.
The site is not easy to identifiable \& accessible from search Contact us information No information to communication with company.

Help-customer service Customer service is easy to find; searching for help Compatibility site works with different browsers Foreign language \&currency The site no different language.

\section{Design}

Use a very simple aesthetic design. Use appropriate images. Font types are easy to read \& color for both font $\&$ background is appropriate. Appropriate page design

\section{Transaction:-}

Easy order process Easy to registration in the site ;but not easy to changing the content of the shopping cart 
Ordering information No payment option\& no return and Content: Up-to-date, Relevant and Accurate information, refund policy

also the site has Information about the company and the

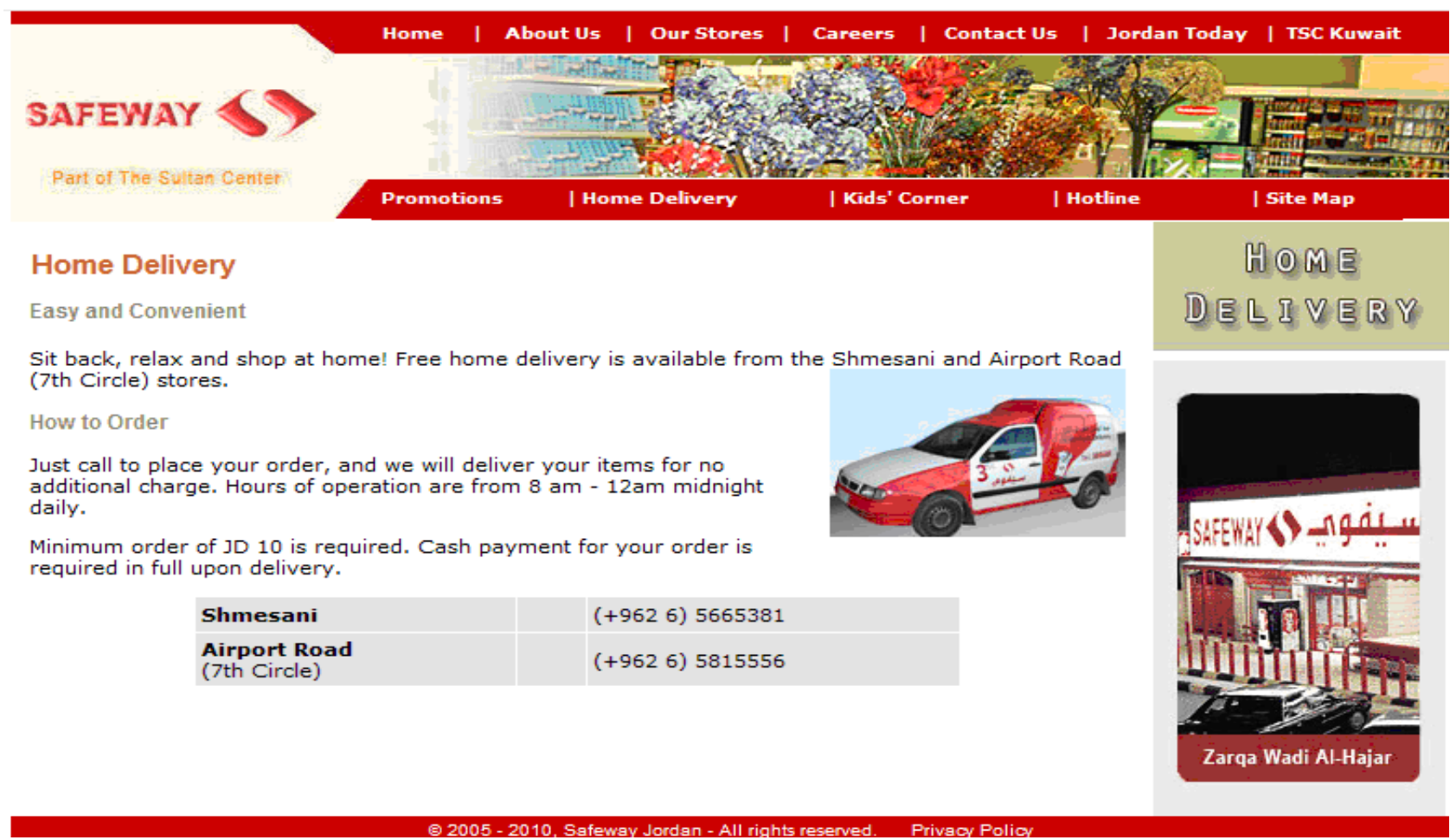

Fig-3 Safeway website

Delivery information Delivery time ; cost ; areas \& product. address

Accessibility and communication: Easy to find and Order-delivery Company not inform the customer about access web site order status

Reasonable security\& privacy Not use secure in this site

\section{NEW WEBSITE}

3- the last site in this research is a mall website in Jordan that represents many activities which contain home delivery the address of the site is : about every item in the website.

http: Ilwww.safeway.com.jo

\section{Architecture and navigations:}

Consistency: red color keys, the background Color white, treated.

font size $=16$, There is great consistency between the colors because of the color red Intro and the end of the site Navigation support: have an index but complex and does not have a search function.

Researchers focus on the security problem by adding a flag for each record to prevent any person with bad history from using the site.

Working links are very clear, good working, resourceful Also we use registration procedure and keep phone links and don't have external links.

Orphan page: site has many orphan pages.

Logical structure of site: understand people easily (usability) but we think developers must reduce the writings and images of more than simple navigation menu numbers to prevent the tricks and thrift.

Search in our website use optimal algorithms to get the results in short time and give the user many options to make the search in simple navigation menu.

Site doesn't have any orphan page and links are used. Figure 4 show the first page of devtohome website. 


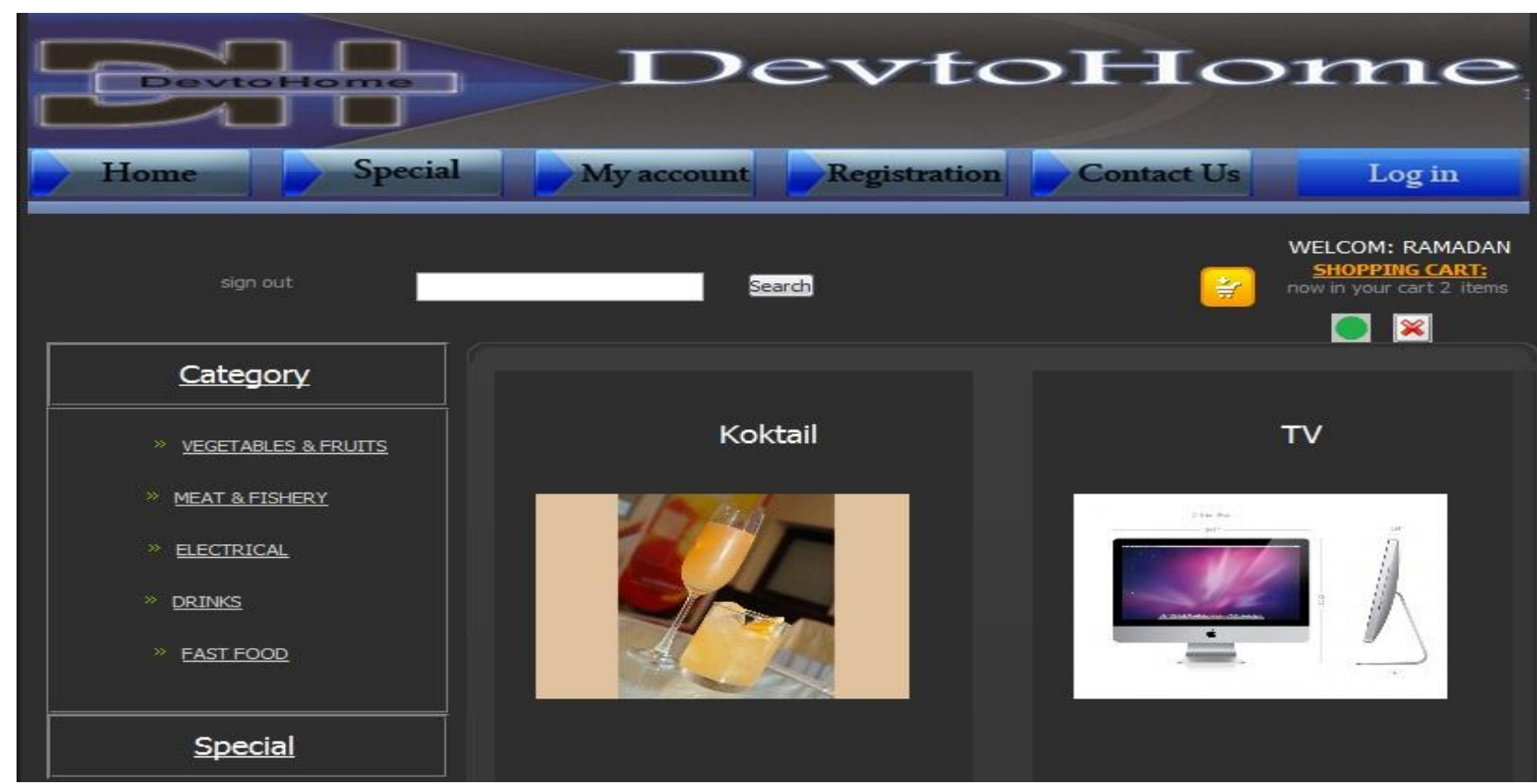

Fig-4 New Designed Website (Devtohome )

\section{CONCLUSION AND FUTURE WORK}

In this research, a comparison of ten popular sites related to e-marketing, and home delivery were adopted. More than thirty factors affecting the websites design were used. Research team took advantages and disadvantages from these sites to present a new site for home delivery marketing.

Research team hope to design a questionnaire, to assess new site performance and to compare with other predecessor sites.

\section{ACKNOWLEDGEMENT}

Researchers thank a lot for all persons and agencies who help to achieve this research.

\section{REFERENCES}

[1] Kotler, P. \& Anderson, A. , Strategic Marketing For Non Profit Organization, $5^{\text {th }}$ Ed. , Practice - Hall , N.J.,1996.

[2] Rob Stokes and mind quirk, e-marketing the essential guide to online marketing, $2^{\text {nd }}$, Rob stokes.

[3] Aladwani, A. and Palvia, P. (2002), Developing and validating an instrument for measuring user-perceived web quality, Information \& Management, Vol. 39 No. 6, pp. 467-76.

[4] Bakos, J.Y. (1991), A strategic analysis of electronic marketplaces, MIS Quarterly, Vol. 15 No. 3, pp. 295-310.

[5] Banks, D. (1997), "What clicks?", Wall Street Journal, Vol. 229 No. 55, pp. R1-R4.

[6] Barnes, S. and Vidgen, R. (2001), An evaluation of cyberbookshops: the Web Qual method, State University of New York at Buffalo, NY, USA , ISBN 0-542-11771-1, (C2005.

[7] Guelcin Bueyuekoezkan , Evaluating Government Websites Based on a Fuzzy Multiple Criteria Decision-Making Approach, journal Article, Int. J. Uncertainty Fuzziness Knowl.-Based Syst, PP 321343 ,Volume 15 issue 3, 2007.

[8] Catalin Bocanu, A Comparison Between Static and Dynamic Websites, September 5th, 2007.

[9] Catalin Bocanu, Instant Creation of a Real Estate Website with Joomla, September 5th, 2007, visited 16/3/2011,Website:http://news.softpedia.com/news/InstantCreation-of-a-Real-Estate-Website-with-Joomla-63090.shtml
[10] Gary P. Schneider, Electronic Commerce, Course Technology, A division of Thomson Learning Inc, Fourth Annual Edition, 2003, ISBN 0-619-15955-3.

[11] Efraim Turban, David king , Jae Lee and Dennis Viewand, Electronic Commerce a Managerial Perspective , Person Education , 2004, ISBN: 0-13-123015-8.

[12] Mattkew Reynolds, Beginning E-Commercewith vb, SQL Server 7 \& MTS, work Press: 2000, ISBN: 1-861003-98-6.

[13] Tyler Moore and Richard Clayton , Evaluating the Wisdom of Crowds in Assessing Phishing Websites, Financial Cryptography and Data Security (FC) 2008, LNCS 5143, pp. 16-30.

[14] Baker College Online Library Document, (2008), EVALUATING WEB SOURCES, Baker College Online Library,

\section{BIOGRAPHY}

Dr. Mohammed Al Refai Chairman of Software Engineering in Zarqa university, Jordan.

Mhmmad Al_sonbati, Sawsan Houdaly, Ghadeer Mallah, graduated students in Software Engineering in Zarqa university, Jordan. 